\title{
MULTI-OBJECTIVE GENETIC OPTIMIZATION FOR SCHEDULING A MULTI-STOREY BUILDING
}

\author{
Fatma A. Agrama
}

\author{
Civil Engineering Department, Menofia University, Egypt, E-mail: fatmaagrama@ hotmail.com
}

\begin{abstract}
Multi-objective optimization problems can be found in various fields, however, optimal decisions need to be taken in the presence of trade-offs between two or more conflicting objectives. This paper proposes developed Genetic Algorithms (GAs) enabled multi-objective optimization for scheduling a multi-storey building. However, a trade-off between time and cost for habitation projects is required to face limited fund and increasing population environment. This problem has an important position for developing countries' governments because it's related to low cost habitation projects. Multi-storey buildings are classified as special repetitive projects because of skeleton constraints. Activities can be classified into: repetitive and non-repetitive ones. The present model enables construction planners to direct controlled time-cost construction plan by investigating optimal plans, generated from a set of feasible alternatives, which minimize project duration, total number of crews, and total work interruptions. This model satisfies the following aspects: 1) it is based on the line of balance technique; 2) it considers non-serial typical activities networks with finish-start relationship and both lag or overlap time between activities is allowed; 3 ) it utilizes a tool to identify activities in a typical repeated floor; 4) it is developed as a spreadsheet template that is easy to use. Details of the model with visual charts are presented. An application example is analyzed to illustrate the use of the model and demonstrate its capabilities in optimizing the scheduling of a multistorey building.
\end{abstract}

Keywords: Construction Management, Multi-storey building, Line of balance technique, Genetic optimization.

\section{INTRODUCTION}

Developing countries face an increase in population, so, the habitation problem is considered one of their priorities. A lot of efforts have been exerted to solve this problem either for construction systems or for their management. Innovated construction systems were introduced to make time/cost improvements. However, construction systems influence the management of habitation projects which needs complete description of their characteristics. One systematic approach to elaborate theoretical characteristics and components of habitation projects process was produced (Hashem, 2007). Another framework of low-income housing to estimate the time and cost required for construction was produced as well (Marzouk et al., 2010).

Multi-storey building projects are characterized by the repetitiveness of typical units. Network techniques, however, exhibit major drawbacks when applied to schedule repetitive projects (Reda, 1990; Suhail and Neale, 1994). Alternate graphical methods have been provided a simplistic formulation to maintain crew work continuity for repeated activities through sequential units of such projects. The name "vertical 
production method", "time space scheduling method", or "line of balance method" (LOB) any of them is adopted if it is used to schedule vertical repetitive projects (O'Brien, 1975; Stradal and Cacha, 1982). However, these methods are mainly graphical based techniques which limit their practical use. Although graphical methods are not adequate in scheduling repetitive projects, they are the familiar one which represents a start point for various inspired mathematical research-based techniques since the 1960s.

There have been sequential mathematical efforts to identify and treat problems associated with scheduling and optimizing linear projects (Ammar, 2013; Hegazy et al., 1993; Liu and Wang, 2012). However, scheduling of vertical repetitive works is usually handled through heuristic methods (Kang et al., 2001) or knowledge based systems (Thabet and Beliveau, 1994). Generally, the traditional optimization techniques do not guarantee a global optimum solution and may be trapped in local optima in case of large-scale problems (Goldberg, 1989). Optimization models for linear construction projects often present a single objective that either minimizes the projects time or cost (Liu and Wang, 2012). There is a need for advanced models that can help construction planners to generate optimal construction schedule that satisfies the specific requirements with respect to time and cost of the construction project being considered. LOB-based multi-objective optimizations were developed but for scheduling linear construction projects (Hyari and El-Rayes, 2006; Senouci and Al-Derham, 2008).

The scope of this paper is to modify the developed multi-objective optimization model for scheduling linear construction projects (Agrama, 2012) to adopt for scheduling a multi-storey building. The model enables construction planners to generate, from a set of feasible alternatives, optimal/ near-optimal construction plans that minimize project duration, number of synchronized crews, and crew work interruptions.

\section{SCHEDULING REPRESENTATION}

Scheduling representation of a multi-storey building must be proposed. Figure 1 shows the form of LOB scheduling technique activities, where activities are plotted with the axes being units versus time. The model is organized as:

1. Units are typical floors which repeated vertically;

2. The type of activity must be identified to: non-repetitive ones (foundation and roof) and repetitive ones (skeleton and non-skeleton either working upward or downward).

- Foundation activities which will be carried out before execution of the first repetitive unit such as; excavation and footings,

- Roof activities that will be carried out only at the last floor such as; roof insulation,

- Skeleton activities which will be repeated at sequential floors but cannot start at an upper floor before all skeleton activities at a lower floor are finished, i.e. skeleton constraint, such as: columns, beams, and slabs in traditional construction system,

- Non-skeleton activities, which will be repeated at sequential floors, and they are classified into two categories depending on the workflow direction: upward or 
downward. Brickworks is an upward non-skeleton activity. However, finishing external walls is a downward non-skeleton activity.

\section{The model considerations are:}

- Non-serial activities' network with multi-beginning and/or multi-finishing activities is permitted.

- Relationship between activities is finish to start and either positive or negative lags are permitted.

- Each repetitive activity is considered with corresponding typical duration and number of available crews.

- To finish external walls as a downward non-skeleton activity, only one crew is used, crews must not work at succeeding floors in the same face of the building. However to speed this type of activity, it is mandatory to divide work according to number of building faces that each face may be carried out by one crew.

\section{MODEL FORMULATION}

To simplify the modeling task and present a model in a format that is customary to practitioners, a spreadsheet tool, open software Excel, is used for implementation. The spreadsheets have been proven suitable as a tool for developing computerized models, such as the one at hand. The spreadsheet formulation for this model is carried in the following subsections on the case study project described in the literature [Agrama 2006 ] to validate the model. The project consists of five-storey building each has a $24^{\text {th }}$ activities network, Figure 2. The spreadsheet model that comprises activities' data is shown in Figure 3, with the model details provided in the next subsections along with the description of the calculations.

\section{Crew Synchronization Calculations}

One objective of the model is to find the minimum combination of synchronized crews which satisfies the available number of crews $C_{v}(i)$ for each activity $i$. The additional number of crews $C_{d}(i)$ is a variable which ranges from a single crew to the available limit $C_{v}(i)$. This item is handled for upward non-skeleton activities; however, other activities have only one crew limitation. Foundation and roof activities are nonrepetitive activities and hence they need not more than one crew. A downward nonskeleton activity has special treatment, which prevents using more than one crew. A skeleton activity at a floor waits until all skeleton ones at the lower floor to finish, so it needs no more than one crew, see Figure 1. The initial calculation of the activity's progress rate without crew-work interruption, based on LOB scheduling stated at (Hegazy et al., 1993), must be based on only one crew. The initial progress rates of nonskeleton repetitive activities that must be negative for downward ones $(w)$ and positive for the upward ones $(e)$ are represented by Eq.(1).

$$
R_{0}(i)= \pm 1 / D(i) \quad i \in(e, w), i=1,2, \ldots, I
$$

where; $e$ and $w$ are upward and downward non-skeleton activities, respectively. Total number of activities $(I)$ is the summation of number of foundation activities $(F)$, 
number of skeleton activities $(K)$, number of upward non-skeleton ones $(E)$, number of downward non-skeleton ones $(W)$, and number of roof ones $(O)$.

Upward non-skeleton activity $(e)$ that may have more than one crew requires further examination to determine whether it is worth using some or all of the available ones. The additional crews $C_{d}(e)$ is an integer variable that must be less than the available limit, Eq.(2). The use of additional crews $C_{d}(e)$ is allowed only to upward non-skeleton activities that have a slower progress rate than their predecessors or those have no predecessors; thus speeding those slow activities and thereby reducing project duration. The initial progress rate of an upward non-skeleton activity $R_{0}(e)$ which has predecessors is compared with the real progress rate of its predecessors $R_{r}(p)$, will illustrate next, to determine the crew multiplier variable $\mu l(e)$ of zero-one value for each upward activity to signal whether the activity is allowed or disallowed to have additional crews, Eq. (3). The non predecessors' upward activity(ies) may be speeded if it(they) has(have) more available crews. Figure 4 illustrates the cases of upward nonskeleton activity (ies) that has (have) foundation, roof, skeleton, or downward nonskeleton predecessors whenever speeding may positively affect the total project duration, Eq.(3). Then the actual number of crews $C_{a}(e)$ can be calculated by adding the allowed additional crews to the initial crew as given by Eq.(4). Consequently, the actual progress rate $R_{a}(e)$ of each upward activity needs to be recalculated based on the actual number of crews using Eq.(5). Whereas, the actual progress rate $R_{a}(w)$ of each downward activity is still as the initial one.

$$
\begin{array}{ll}
C_{d}(e)<C_{v}(e) ; \text { integer } & e=1,2, \ldots \ldots, E \\
\mu l(e) \in(0,1) ; \mu l(e)=1 & \text { If } R_{0}(e)<\operatorname{MIN}\left\{R_{r}(p)\right\}, p \in(k, e) ; \\
& \text { Or if } p \in(f, o, w) ; p=1,2, \ldots \ldots, P(e), P(e) \neq 0 ; \\
& \text { Or if } \mathrm{P}(\mathrm{e})=0
\end{array}
$$

Where; $P(e)$ is the total number of predecessors of activity $e$.

$$
\begin{array}{ll}
C_{a}(e)=1+\mu 1(e) \times C_{d}(e) & e=1,2, \ldots \ldots, E \\
R_{a}(e)=C_{a}(e) / D(e) & e=1,2, \ldots \ldots, E
\end{array}
$$

If the upward non-skeleton activity needs to be interrupted to minimize total project duration, its progress rate tends to be slower. The real progress rate of predecessors $R_{r}(p)$ is used to express the activity progress rate through units including actual interruptions, see Figure 4-e.

$$
R_{r}(p)=(N-1) /[S(p, N)-S(p, 1)] \quad p \in(k, e)
$$

Where; $S(p, N)$ and $S(p, 1)$ are the start times of predecessor $p$ at the last unit $N$ and first one, respectively.

The calculations of crew synchronization for each activity are shown in Figure 3 (columns $\mathrm{P}$ to $\mathrm{U}$ ). Column $\mathrm{P}$ represents the activity crews' limitation, which must not exceed total number of repetitive units for upward non-skeleton activities and one crew for the other activities. Column Q is direct application of Eq.(1). Column R represents the independent variable, number of additional crews $C_{d}(i)$, to be utilized for each 
activity; while column $\mathrm{S}$ is the formulation described by Eq.(3) for allowing/disallowing additional crews. The actual number of crews to be used for each activity $i$ is adjusted in column T considering crew availability for each kind of activities, Eq.(4). Accordingly, the actual progress rate is calculated in column U, Eq.(5). For example, the thirteenth activity which has an additional crew variable equals to one crew (column $\mathrm{R}$ ) is permitted by five available crews (column P) and crew multiplier variable (column S) and as such, the actual number of crews for activity $13^{\text {th }}$ is two crews. Conversely, activity ten that has four additional crews is prevented to use all of them because the crew multiplier variable is calculated to be zero.

\section{Interruption Calculations}

Interruptions are permitted only for non-skeleton activities in which they may become beneficial and prevented from being applied to all the others, thus guiding the optimization process as shown in Figure 5. Interruptions become harmful for scheduling if they are applied on downward non-skeleton activities (case c). Skeleton activities are obligatory interrupted to achieve skeleton constraint and it is harmful to allow them more optional interruptions. However, an interruption, in the present study, is defined as the delay of the start of an upward activity $e$ at unit $n$ from its calculated start based on rate of work continuity. An integer independent interruption variable $I N(e, n)$ for activity $e$ at interrupted unit $\mathrm{n}$ is adopted. The use of interruptions is limited only to upward nonskeleton activities with higher progress rates than their predecessors (cases a, b). The actual progress rate of activity $e$ is compared with real progress rates of its predecessors to permit interruption for that activity. Similarly, an interruption multiplier zero-one variable, $\mu 2(e)$, for upward activity $e$ is applied to allow/disallow interruption as given by Eq.(7). However, the upward activity that has roof predecessor is allowed to interrupt (case p). Another zero-one variable $\mu 3(i)$, as well, prevents upward activities with no predecessors, with no successors (case f), or that with either foundation predecessors (case e) or downward non-skeleton predecessors (case g) from having interruptions as given by Eq.(8).

$$
\begin{array}{ll}
\mu 2(e) \in(0,1) ; \mu 2(e)=1 \quad & \text { If } R_{a}(e)>\operatorname{MAX}\left[R_{r}(p)\right] ; p \in(k, e) ; \\
& \text { Or If } p \in o ; p=1,2, \ldots \ldots, P(e) ; P(e) \neq 0 \\
& e=1,2, \ldots \ldots, E ; \operatorname{Sc}(e) \neq 0 \\
& \text { If } P(e) \neq 0 ; p \notin(\mathrm{f}, w) ; \text { Or } S c(e) \neq 0 ; \\
& e=1,2, \ldots \ldots, E
\end{array}
$$

where; $S c(e)$ is total number of successors of activity $e$.

If interruption is permitted the actual interruption will equal the independent interruption variable and if interruption is prevented (i.e. $\mu 2(i)$ or $\mu 3(i)$ is zero) the actual interruption is zero, Eq.(9). However, the actual interruption value of activity $e$ at first unit must be eliminated, Eq.(10). Allowing/disallowing interruptions within maximum limitation is represented in columns $\mathrm{V}$ and $\mathrm{W}$, Figure 3. Column $\mathrm{V}$ is direct application of Eq.(8), while Eq.(7) is represented in column W.

$$
\begin{array}{ll}
I N_{a}(e, n)=I N(e, n) \times \mu 2(e) \times \mu 3(e) & e=1,2, \ldots, E ; n=2,3, \ldots, N \\
I N_{a}(e, 1)=0 & e=1,2, \ldots, E
\end{array}
$$




\section{Schedule Calculations}

Once the no predecessors' activities in the first unit start at time zero, Eq.(11), there are another three constraints control calculations of start time $S(i, n)$ of an activity $i$ at unit $n$ :

$S(i, 1)=0 \quad i \notin(o, w) ; i=1,2, \ldots, I ; P(i)=0$

First. is the LOB considerations that guarantee crew synchronization movement $R_{a}(i)$ and interruption consideration $I N_{a}(i, n)$ of non-skeleton activity $(i \in e, w)$ at unit $n$, Eq.(12).

$S(i, n)=S(i, n-1)+1 / R_{a}(i)+I N_{a}(i, n) \quad i \in(e, w) ; n=2,3, \ldots \ldots, N$

Where; $1 / \mathrm{R}_{a}(\mathrm{i})$ term is the amount of shift time required to achieve crew synchronization and $I N_{a}(i, n)$ term is the actual interruption time for activity $i$ at unit n, when $i \in e$ only, interruption must be eliminated for downward activity.

Second. is the logical relationship consideration that guarantees complete finishing of all predecessors before activity start. This constraint must be considered at all units for all types of activities. Nevertheless, this constraint is applied only at first unit for foundation activities and at last unit for roof activities, Eq.(13).

$$
\begin{aligned}
S(i, n) \geq \operatorname{MAX}[S(p, n)+D(p)+L(i, p)] & p=1,2, \ldots \ldots, P(i) ; P(i) \neq 0 ; \\
& \text { If } i \in \text { f then } n=1 ; \\
& \text { If } i \in O \text { then } n=N ; \\
& \text { If } i \in(k, e, w) \text { then } n=1,2, \ldots \ldots, N
\end{aligned}
$$

Where; $[S(p, n)+D(p)]$ term represents finish time of predecessor $p$ at unit $n$ and $L(i, p)$ is the lag between activity $i$ and its predecessor $p$.

Third. is the skeleton constraint that is applied only for skeleton activities to guarantee complete finishing of all skeleton activities at a lower floor before any skeleton activity $k$ at an upper one starts, Eq.(14). The finish time $F(i, n)$ of an activity $i$ at unit $n$ can be calculated using Eq.(15) once start time is calculated.

$S(k, n) \geq \operatorname{MAX}[S(K, n-1)+D(K)] \quad k=1,2, \ldots, K ; n=2,3, \ldots \ldots, N$

Where; $\operatorname{MAX}[S(K, n-1)+D(K)]$ term represents finish time of the last skeleton activity(ies) $\mathrm{K}$ at unit $\mathrm{n}-1$.

$$
\begin{aligned}
F(i, n)=S(i, n)+D(i) \quad & i=1,2, \ldots, I \\
& \text { If } i \in \text { f then } n=1 ; \\
& \text { If } i \in O \text { then } n=N ; \\
& \text { If } i \in(k, e, w) \text { then } n=1,2, \ldots \ldots, N
\end{aligned}
$$

The start time at first unit of non-skeleton activity $i \in e, w$; which has predecessors, must be adjusted first on all possible paths in order to prevent a conflict in the logical relationship. The maximum of start times $S(i, n)$ of activity $i \in e, w$ according to the predecessors figures at unit $\mathrm{n}$ are considered to adjust start time of the same activity at 
first unit maintaining LOB considerations of the activity, Eq.(16), see Figures 6 and 7. In these figures, a dashed line parallel to the right side of the predecessor $p$ represents the limit respects logical relationship consideration. This limit must not be violated by starting of any successor, first term of Eq.(16). However, to start activity $i \in e, w$ at first unit, slope of activity $i$ must be respected by parallel lines down from that limit at each unit to the first one then the summation of activity interruptions up to that unit is subtracted, second term of Eq.(16). Thus; constituting many start times of activity $i$ at first unit. The maximum of them is the adjusted start time of activity $i$ at first unit which respects the two considerations.

$$
\begin{aligned}
S(i, 1)=M A X\{[F(p, n)+L(i, p)]-[ & \left.\left.(n-1) / R_{a}(i)+\sum_{1}^{n} I N_{a}(i, n)\right]\right\} \\
& \text { If } p \in f \text { then } n=1 ; \\
& \text { If } p \in \text { o then } n=N ; \\
& \text { If } p \in(k, e, w) \text { then } n=1,2, \ldots \ldots, N \\
& i \in(e, w) ; i=1,2, \ldots \ldots, I ; \\
& p=1,2, \ldots, P(i) ; P(i) \neq 0
\end{aligned}
$$

where; $[F(p, n)+L(i, p)]$ term guarantees the completely finishing of predecessor $p$; $L(i, p)$ is the lag between activity $i$ and its predecessor $p$ and $\left[(n-1) / R_{a}(i)+\right.$ $\left.\sum_{1}^{n} I N_{a}(i, n)\right]$ term maintains LOB considerations through sequential units up to unit under consideration, considering that downward activities are prevented to interrupt.

Schedule calculations, at the spreadsheet, for all activities at each unit are set up in columns at the right of column W, see Figure 8. For each unit, columns are set up to calculate start and finish times of all activities. Another column is also included to specify the start time of the activity at first unit Eq.(16), considering the maximum value of $S(i, 1)$ up to unit under consideration. Other columns are also included to specify the interruption values at all units. For the example at hand, upward activity $13^{\text {th }}$ in the third unit which has an interruption variable of two days is disallowed from having interruption and as such, the actual interruption used is zero.

\section{OPTIMIZATION IMPLEMENTATION}

Many evolutionary algorithms have been developed for multi-objective optimization problems. Traditional optimization techniques have difficult to be extended to the multiobjective case. Genetic algorithms are search and optimization tool that assist decision makers to identify optimal or near-optimal solutions for problems with large search space. They are inspired by the mechanics of evolution that they adopt the survival of the fittest; the structured exchange of genetic materials among population members over successive generations as a basic mechanism for the search process (Goldberg, 1989). To obtain satisfactory compromise solutions, some algorithms convert multi-objective optimization problem to a single optimization one. The weighted-sum method is a kind of these algorithms that combined with genetic algorithms method to optimize the present model (El-Shorbagy, 2010). 
Once the model was created, optimization was carried out by one of the available commercially software for GAs optimization. Evolver 4.0 works as an add-on program to the Microsoft Excel environment and as such, suits the present model. Evolver does not need to know the "nuts and bolts" of the GAs optimization theory (Evolver Manual, 1998). Evolver has a user friendly interface and facilitates user specification of the optimization parameters. The string size is determined by the model, considering the total number of independent variables included in the analyzed project, illustrated next. The population size, for matting pool, is estimated based on the string size in order to improve the quality of the solution. The crossover rate represents the probability that two strings will swap their bits creating new offspring strings that consist of parts taken from selected parent strings. The mutation rate represents the probability that a bit within a string will be flipped (0/1variation) introducing random changes in the solution population. The model involved the setup of the multi-objective function, independent variables, and operating environmental constraints.

\section{Multi-objective function}

The multi-objective function $M O F$ represented at cell L9, combines three target goals linearly. First is to minimize the project duration PD calculated at cell L7, Eq.17; second is to minimize the total actual crews for all activities, permitted for upward ones only, calculated at cell F7; and third is to minimize the total interruptions for all activities, permitted for upward ones only, at all units calculated at cell F9, see Figure 2. $M O F$ can be computed using planner-specified coefficients' weights that reflect the relative importance of project duration $w_{d}$ at cell S5, summation of synchronized crews $w_{c}$ at cell S7, and summation of work interruptions $w_{i}$ at cell S9 to fit the decision maker. These three coefficients must be positive and sum up to one. Thus, MOF is a summation of different terms that have different units. To solve this drawback, $M O F$ is converted to dimensionless fitness function as shown in Eq.18.

$$
\begin{gathered}
P D=\operatorname{MAX}\left[\begin{array}{ll}
F(i, 1), & i \in w \\
F(i, N), & i \in(k, e)
\end{array}\right] \quad \begin{array}{c}
i=1,2, \ldots \ldots, I \\
\text { Minimize: } M O F=w_{d} \times P D / P D_{1}+w_{c} \times \sum_{i=1}^{l} C_{a}(i) / \sum_{i=1}^{l} C_{v}(i)+w_{i} \times \sum_{i=1}^{l} \sum_{n=2}^{N} I N(i, n) / P D_{1}
\end{array}
\end{gathered}
$$

Where; $P D_{1}$ is the un-optimized project duration and $\sum_{i=1}^{\prime} C_{v}(i)$ is the total available crews for all activities. However, these terms are constant for one project.

\section{Independent Variables}

As mentioned earlier; the independent variables in the present model are the number of additional crews and the interruptions at each unit for upward non-skeleton activities (columns $\mathrm{R}, \mathrm{AB}, \mathrm{AH}, \mathrm{AN}$, and $\mathrm{AT}$ in the present example). The number of variables, as such, is $E+(N-1) \times E$. The $E$ component represents the additional crews vector, while $(N-1) \times E$ component represents a matrix of the interruptions at each unit (except unit 1) of each upward activity. In the present example, ten upward non-skeleton activities and five units result in a total of 50 independent variables. 


\section{Operating Environmental Constraints}

A total of two operating environmental constraints are needed for the model to function properly as follows:

- The additional crews to be utilized must be non-negative integer value and may involve a limit set which less than the number of units, since this method assumes that only one crew works in a single unit. An integer (0-4) is used as a constraint to the number of additional crews of each upward activity.

- Interruption days are limited to integer (0-5) for each unit in each upward activity, for this example.

The optimization screen of Evolver is shown in Figure 9 with the optimization parameters specified. Before running Evolver, all variables were initialized; additional crews and interruptions were set to zeros. Accordingly, the initial project duration $P D_{1}$ was 413 days. The case study was then run on Evolver using a Pentium V 3.6 GHz PC. After running Evolver, an optimum schedule was obtained in 2:30- 5 min (by using a 50 gene population, a crossover value of 0.5 for two types of variables, and mutation values of 0.25 and 0.06 for additional crews and work interruptions variables; respectively). It is noted that once the solution found by Evolver, operations are set to stop if change in last 1000 trials is less than 1\%, was not improving for a complete five minutes of processing; as another stopping condition.

\section{DISCUSSION OF THE RESULTS}

The model was able to significantly reduce the search space by precluding local trapped solutions in the successive generations of the GA. This led to alternative optimal solutions which trade-off among 3-goals: project duration, total synchronized crews, and total work interruptions. Table 1 summarizes some scenarios and shows their impact on schedule performance. It is needed to specify the relative importance of the three goals in the form of three relative weights $\left(w_{d}, w_{c}\right.$, and $\left.w_{i}\right)$. However, three runs for each scenario are done to check stability to optimum goals. MOF is significant value for each scenario, that its values can't be used to compare between different scenarios but to compare between different runs for the same scenario. It is found that the present model is capable of generating the same set of optimal goals for the three runs of scenarios (4-6) at Table 1. The cause of identical optimal goals; may be that those scenarios apply interruption weight $\left(w_{i}\right)$ equals to crews weight $\left(w_{c}\right)$ within decline in the relative importance of project duration weight coefficient $\left(w_{d}=0.7-0.5\right)$. However, more or less values of project duration coefficient can't guarantee stability of optimum goals, scenarios 1-3, 7. Changing interruption weight $\left(w_{i}\right)$ and crews weight $\left(w_{c}\right)$, as scenarios 8, 9; can't guarantee stability of optimum goals, as well. Hence, this model is elastic enough for the planner to choose the priority of goals' weights.

Scenario 1 at Table 1 represents the time optimization case; the same results are obtained by time optimization model of Agrama 2006. Furthermore, the present model is capable of generating the same set of optimal solution as that reported in cost optimization model of Agrama 2006, scenarios 4- 6 at Table 1. The cause of identical optimal solutions, that those scenarios apply interruption and crews weights $\left(\mathrm{w}_{\mathrm{i}}, \mathrm{w}_{\mathrm{c}}\right)$ instead of interruption and crew penalty costs for Agrama model. It is found that the 
present model has not only consistency performance but also introduces an efficient flexible time and cost optimization can be obtained in one model without need to enter so many data for cost as Agrama 2006, that means less implementation effort and time. Another important merit for this model is the benefit of using open software, such Excel, within its versatile features as illustrated next.

\section{LOB Chart}

A LOB chart for one optimum solution (scenario 4- 6, Table 1) using Excel standard chart type, stacked bar, within some implementations is shown in Figure 10. The degree of the LOB details must be carefully evaluated and organized in six individual paths for better understanding, as presented by Agrama, 2012. An experienced scheduler can select a suitable level of detail and scale. The figure shows that $5^{\text {th }}$ and $6^{\text {th }}$ paths are critical. It is expected that foremen and subcontractors will be more receptive to LOB chart than to precedence networks; however, this model can introduce, by Excel, a complete LOB chart not for critical path only.

\section{Further Experimentation}

In addition to the case study presented in this paper, other examples described in the literature were used to validate the model for case of linear projects as well. The simple example by Hegazy and Wassef (2001) then by Agrama (2012) were generated by describing all activities as non-skeleton upward type. The results are identical to those produced in literature. Thus, the present model has not only consistency performance but also introduces an efficient versatile form that:

- Time and flexible cost optimization can be obtained, without need to enter so many data for cost model. That means less implementation effort and time.

- Optimization can be obtained for horizontal or vertical repetition project.

- A complete LOB chart can be introduced, not for critical path only.

\section{SUMMARY AND CONCLUSIONS}

A robust multi-objective optimization model was developed to support the scheduling of a multi-storey building. The model enables construction planners to investigate optimal construction plans that establish optimal trade-offs between project duration, total synchronized crews, and total work interruptions to determine weights which achieve scheduling stability. Each of these plans identifies, from a set of feasible alternatives, optimal crew synchronization for each upward activity and its interruptions at each unit. To accomplish this, the model incorporates: 1) a generalized LOB scheduling formulation at its core for a multi-storey building; 2) non-serial network with typical repeated activities which have finish-start relationship and both overlap and lag times are allowed; 3) crew synchronization within resource constraints; 4) activity interruptions within upper limit; 5) a multi-objective optimization depending on the investigated weighting values of goals is used; 6) GAs technique, Evolver 4.0, has been used in optimization process through add-on Excel spreadsheet as an operating environment; 7) Visual LOB chart for each individual path is presented. An application example of a multi-storey building was analyzed to demonstrate the usefulness of the model and illustrate its capabilities. The results of the optimization conducted prove the 
robustness and consistency. The transparency of the model and its versatile performance hopefully will encourage project managers to utilize it in the planning of repetitive projects. The presented spreadsheet model will be later expanded for a non-typical repeated activity.

\section{REFERENCES}

1. Agrama FA (2006). "The Line of Balance Technique by Genetic Optimization". Doctor of Philosophy Thesis, Tanta Univ., Egypt.

2. Agrama FA (2012). "Multi-objective genetic optimization of linear construction projects". HBRC Elsevier J. 8:144-151.

3. Ammar M, (2013). "LOB and CPM Integrated Method for Scheduling Repetitive Projects". ASCE J. Construction Engineering and Management. 139(1): 44-50.

4. El-Shorbagy MA (2010). "Hybrid Particle Swarm Algorithm for Multi-Objective Optimization". Master of Engineering Thesis. Menoufia Univ. Egypt.

5. Evolver Reference Manual (1998). Palisade Corp. Newfield NY.

6. Goldberg DE (1989). "Genetic Algorithms in Search, Optimization, and Machine Learning”. Reading, Mass: Addison-Wesley Publishing Company.

7. Hashem A (2007). "Housing Management Systems: Theoretical Approach". International Conference towards Low Cost Housing. HBRC. Cairo. Egypt.

8. Hegazy T, Moselhi O, Fazio, P (1993). "BAL: An Algorithm for Scheduling and Control of Linear Projects," Transportation ASCE International. C.8:1-14.

9. Hegazy, T. and Wassef, N. (2001), "Cost Optimization in Projects with Repetitive Nonserial Activities.” ASCE J. Construction Engineering and Management, 127(3): 183-191.

10. Hyari K, El-Rayes K (2006). "Optimal Planning and Scheduling for Repetitive Construction Projects". J. Management in Engineering. 22(1):11-19.

11. Kang LS, Park IIC, Lee BH (2001). "Optimal Schedule Planning for Multiple, Repetitive Construction Process". ASCE J. Construction Engineering and Management. 127(5):382-390.

12. Liu SS, Wang CJ (2012). "Optimization Linear Project Scheduling with MultiSkilled Crews". Elsevier J. Automation in Construction. 24:16-23.

13. Marzouk MM, Omar OA, Abdel Hamid MS, El-Said ME (2010). "An Optimization Algorithm for Simulation-Based Planning of Low-Income Housing Projects". J. Advanced Research. 1:291-300.

14. O’Brien JJ (1975). "VPM Scheduling for High-Rise Buildings". ASCE J. The Construction Division. 101(CO4):895-905.

15. Reda RM (1990). "RPM: Repetitive Project Modeling”. ASCE J. Construction Engineering and Management. 116(2):316-330. 
16. Senouci A, Al-Derham HR (2008). "Genetic Algorithm-Based Multi-Objective Model for Scheduling of Linear Construction Projects". J. Advances in Engineering Software. 39:1023-1028.

17. Stradal O, Cacha J (1982). "Time Space Scheduling Method". ASCE J. Construction Division. 108(CO3):445-457.

18. Suhail SA, Neale RH (1994). "CPM/LOB: New Methodology to integrate CPM and Line of Balance". ASCE J. Construction Engineering and Management. 120(3):667684.

19. Thabet WY. Beliveau YJ (1994). "HVLS: Horizontal and Vertical Logic Scheduling for Multistory Projects". ASCE J. Construction Engineering and Management. 120(4):875-892. 


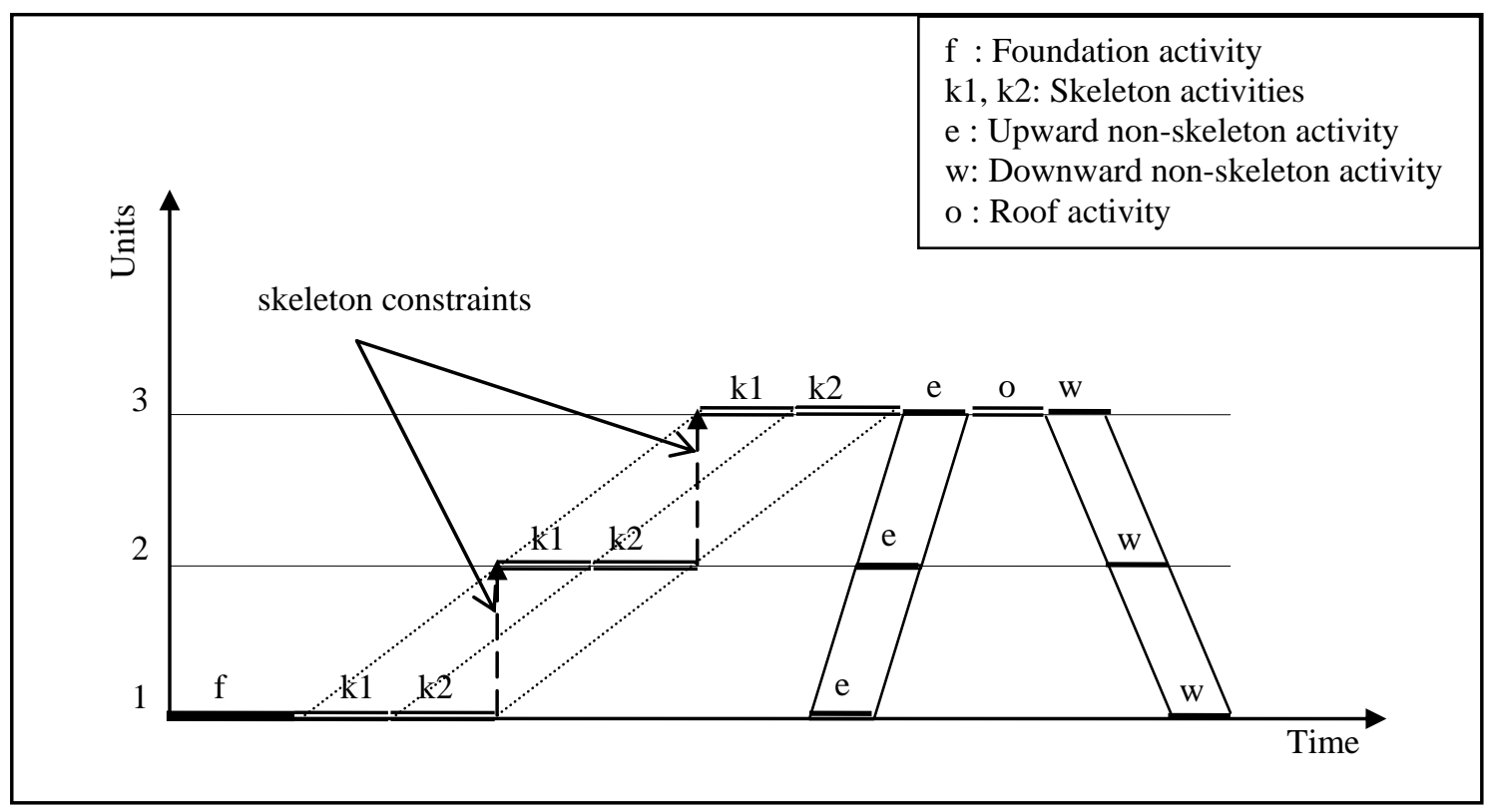

Figure 1. Multi-Storey Building Scheduling Representation

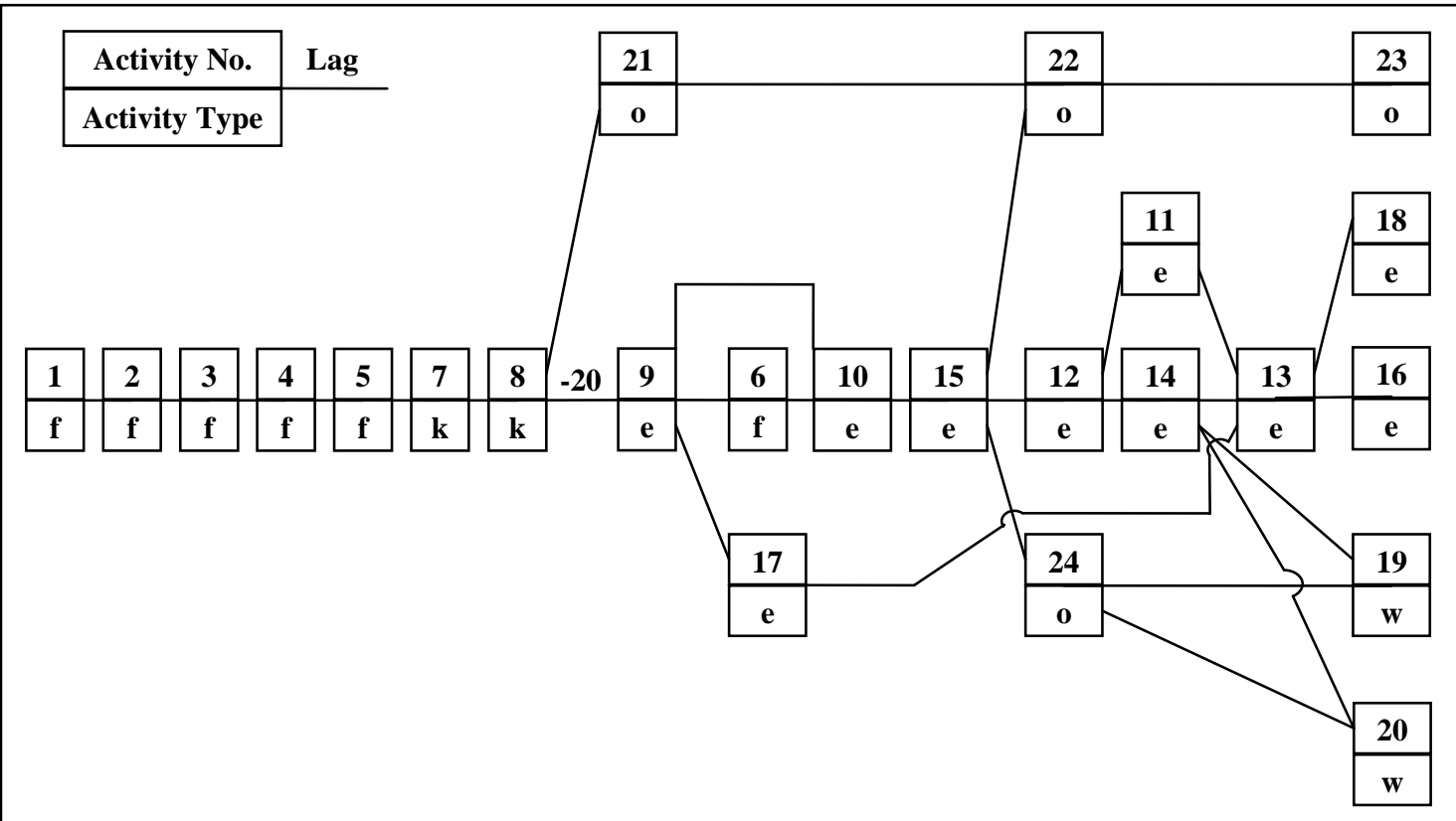

Figure 2. Precedence Network for One Unit of Case Study 


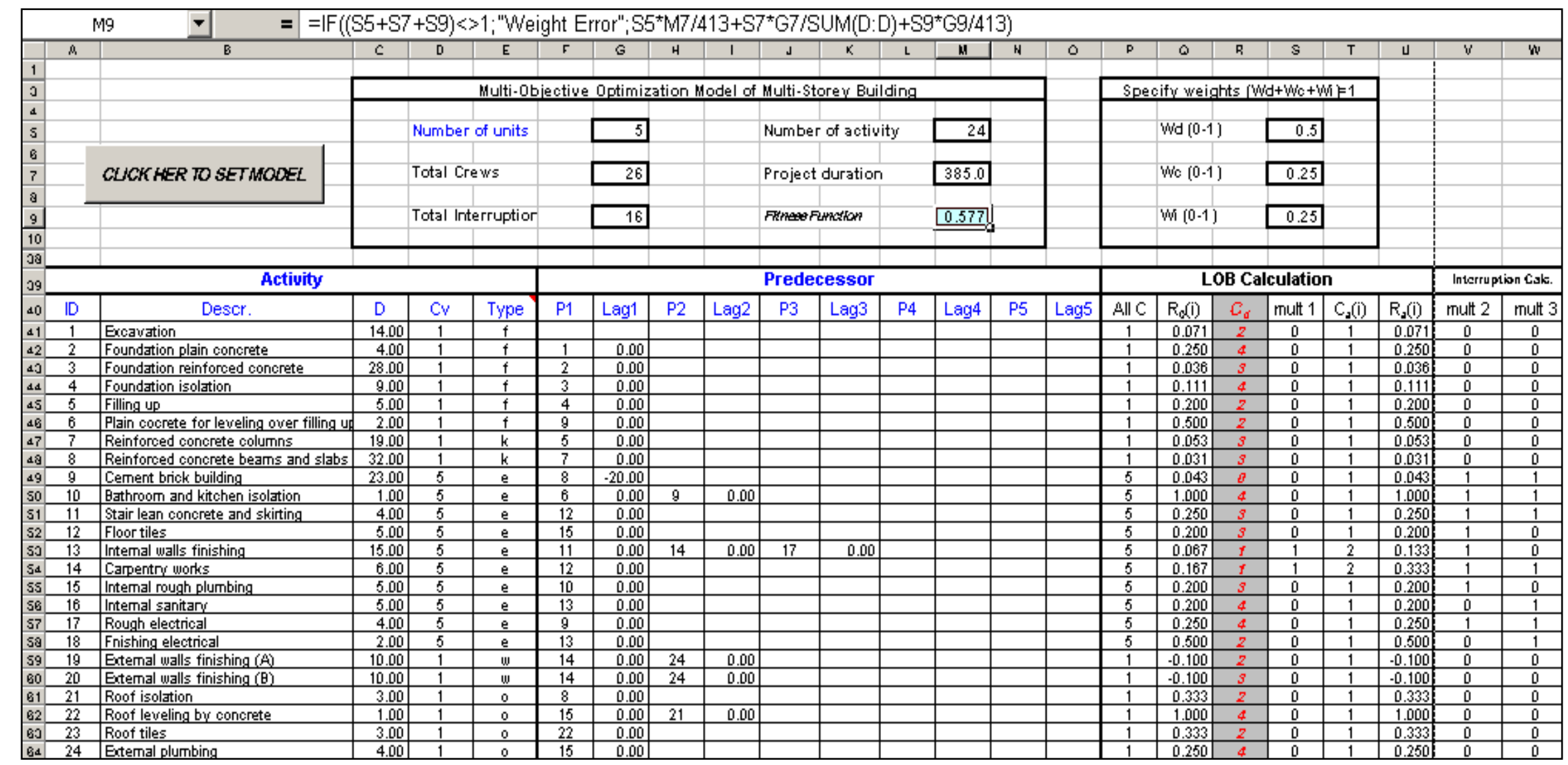

Figure 3. Spreadsheet of Activities' Data, Crew Synchronization and Interruption Calculations 


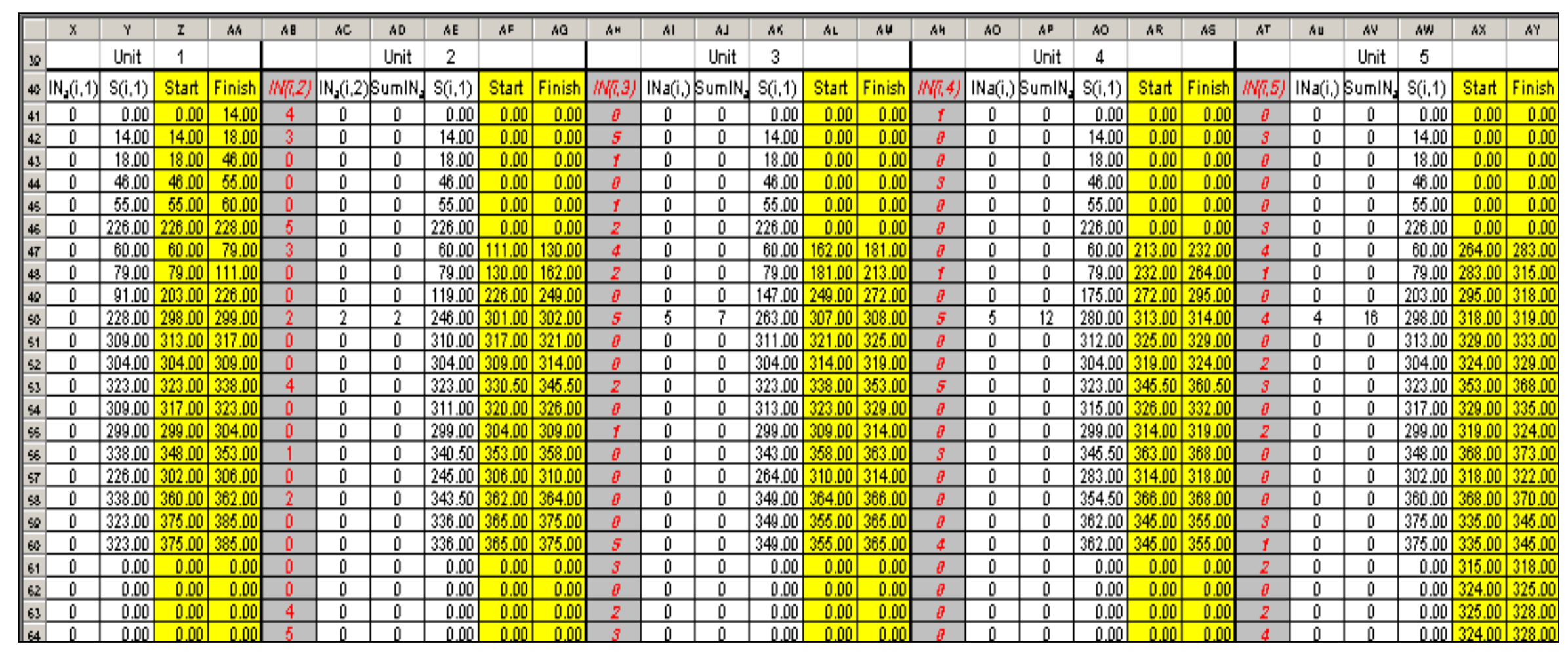

Figure 8. Schedule Calculations after Optimization 


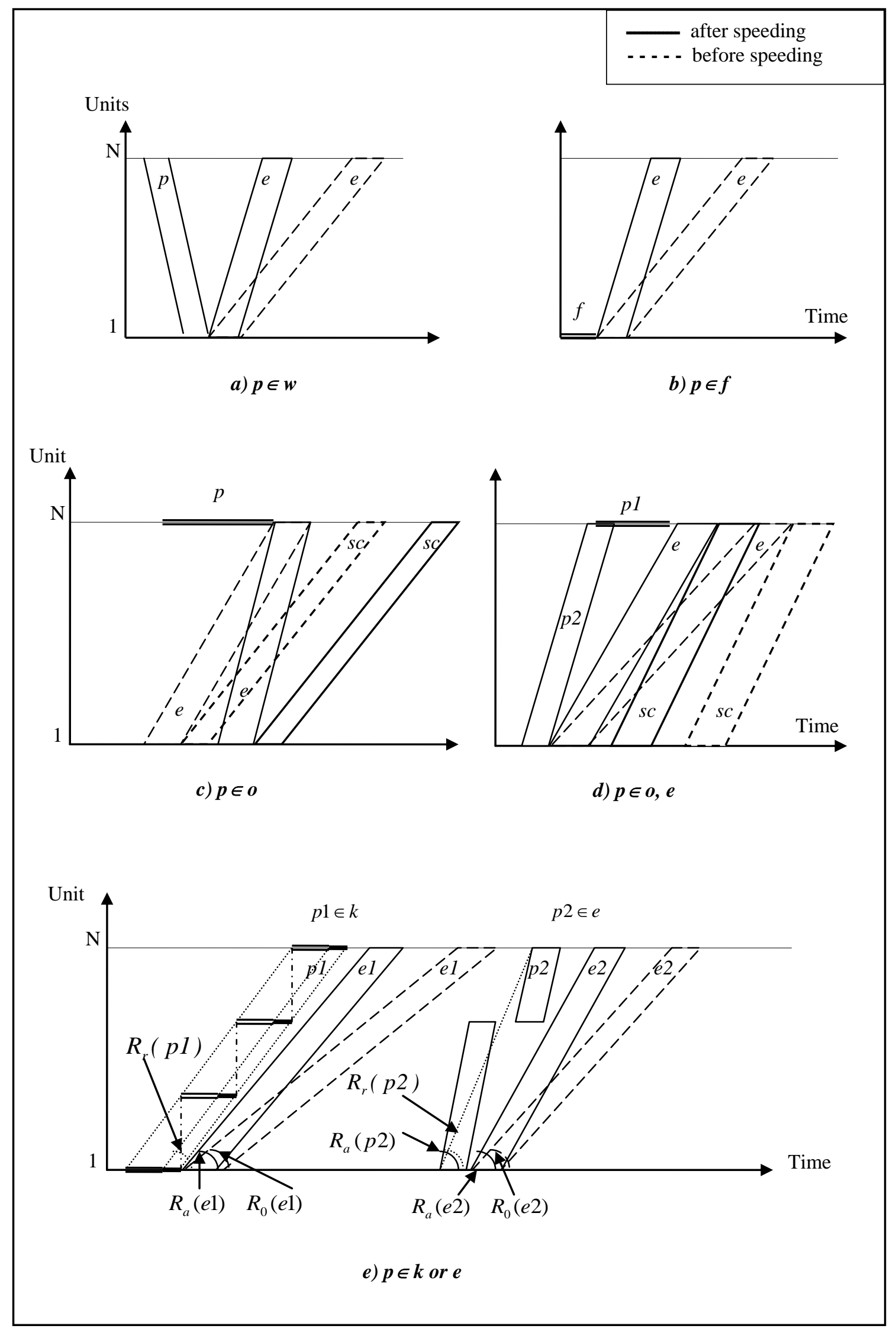

Figure 4. Speeding Cases of Upward Non-skeleton Activities 


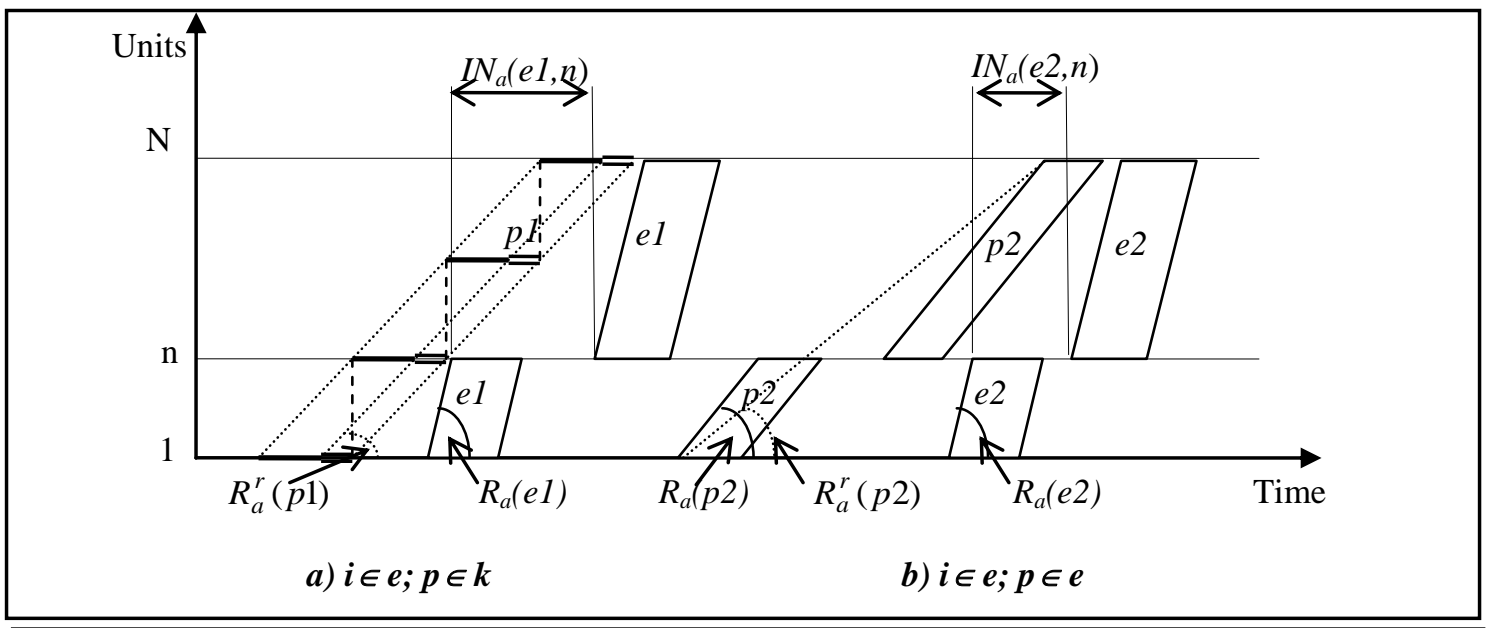

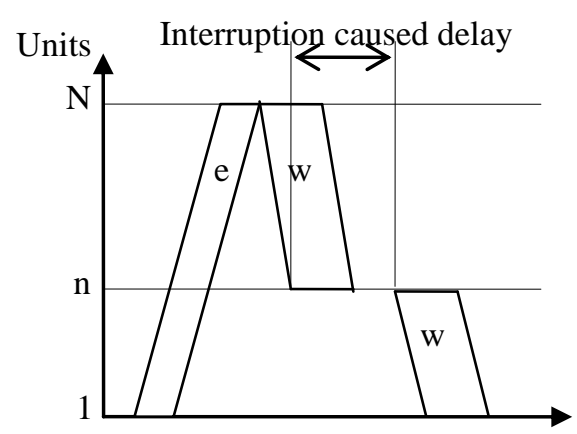

c) $i \in w$

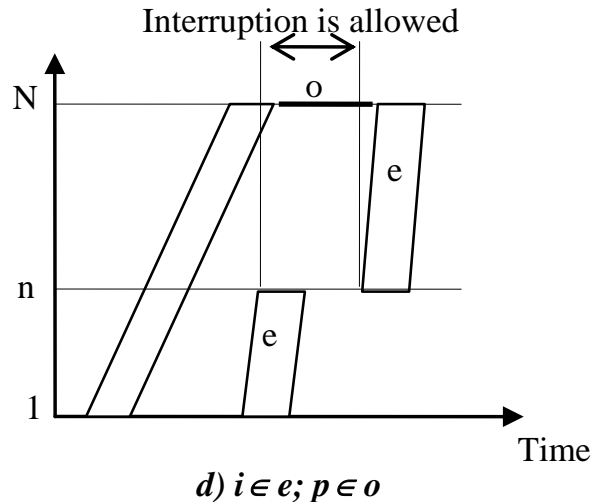

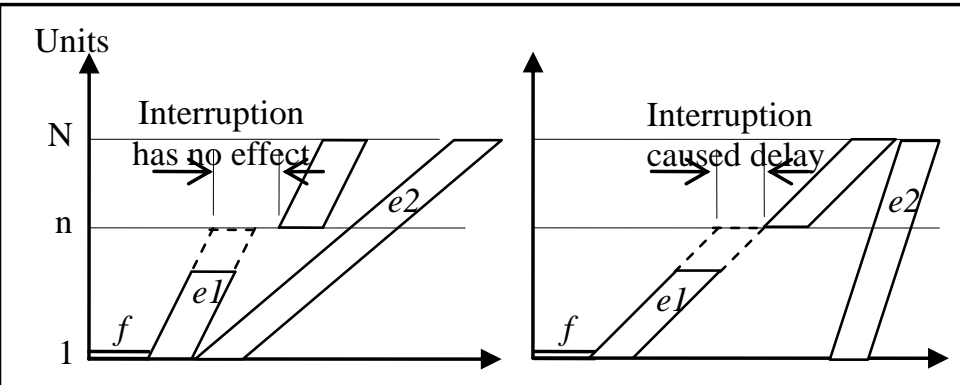

e) $i \in e ; p \in f$

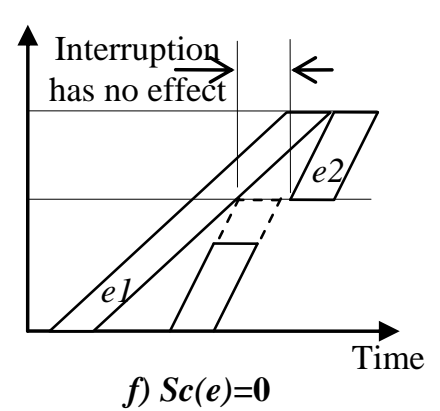

f) $S c(e)=0$

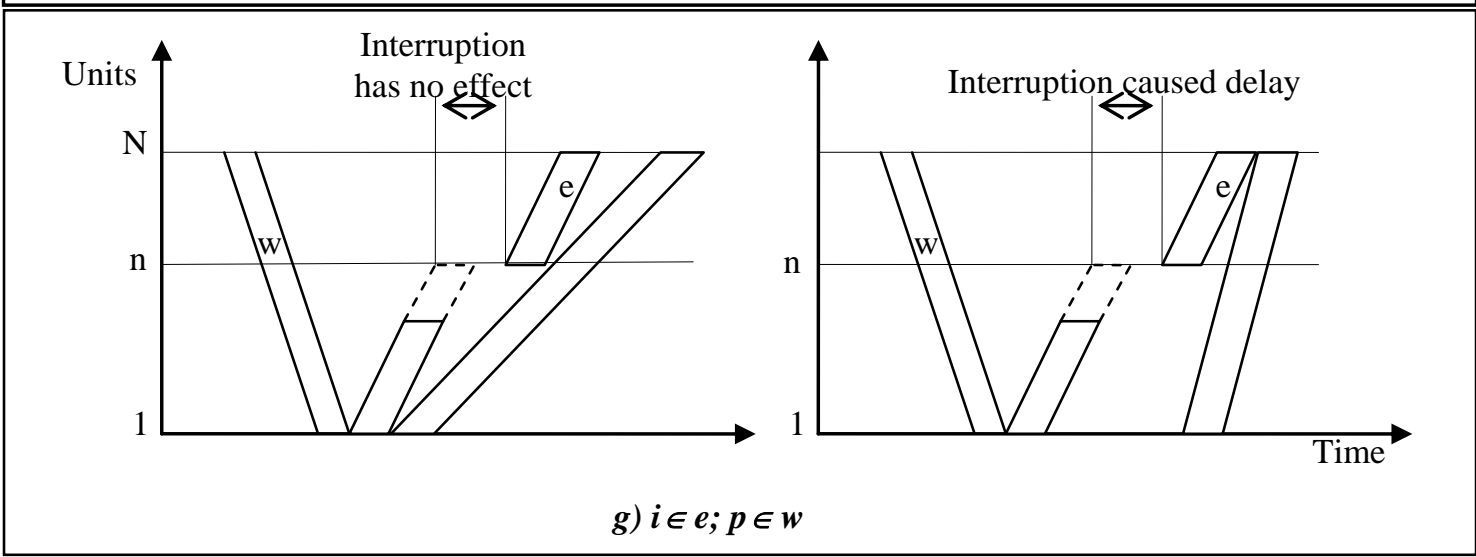

Figure 5. Interruptions Cases of Upward Non-skeleton Activities 


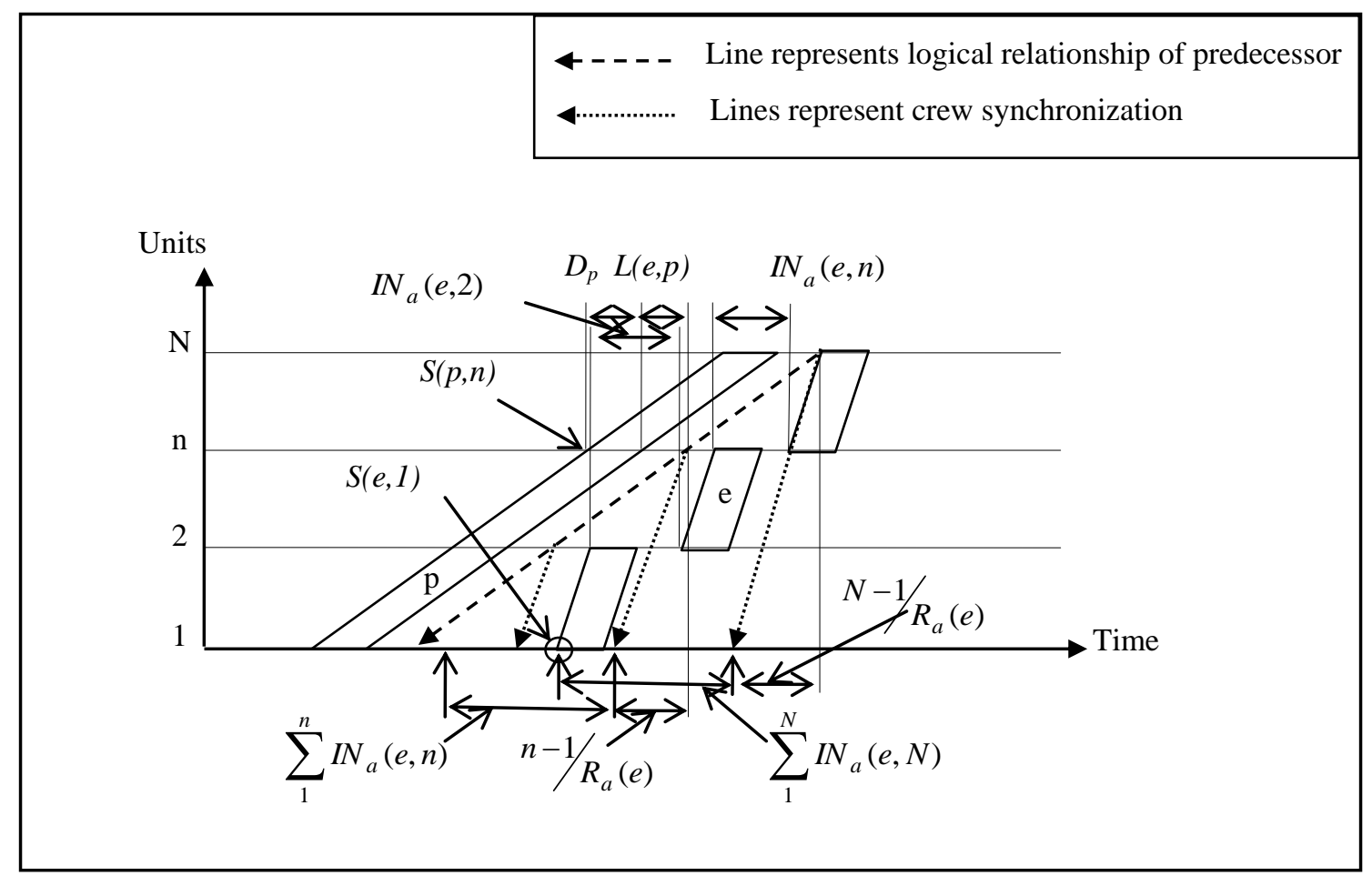

Figure 6. Scheduling Upward Non-skeleton Activities at First Unit

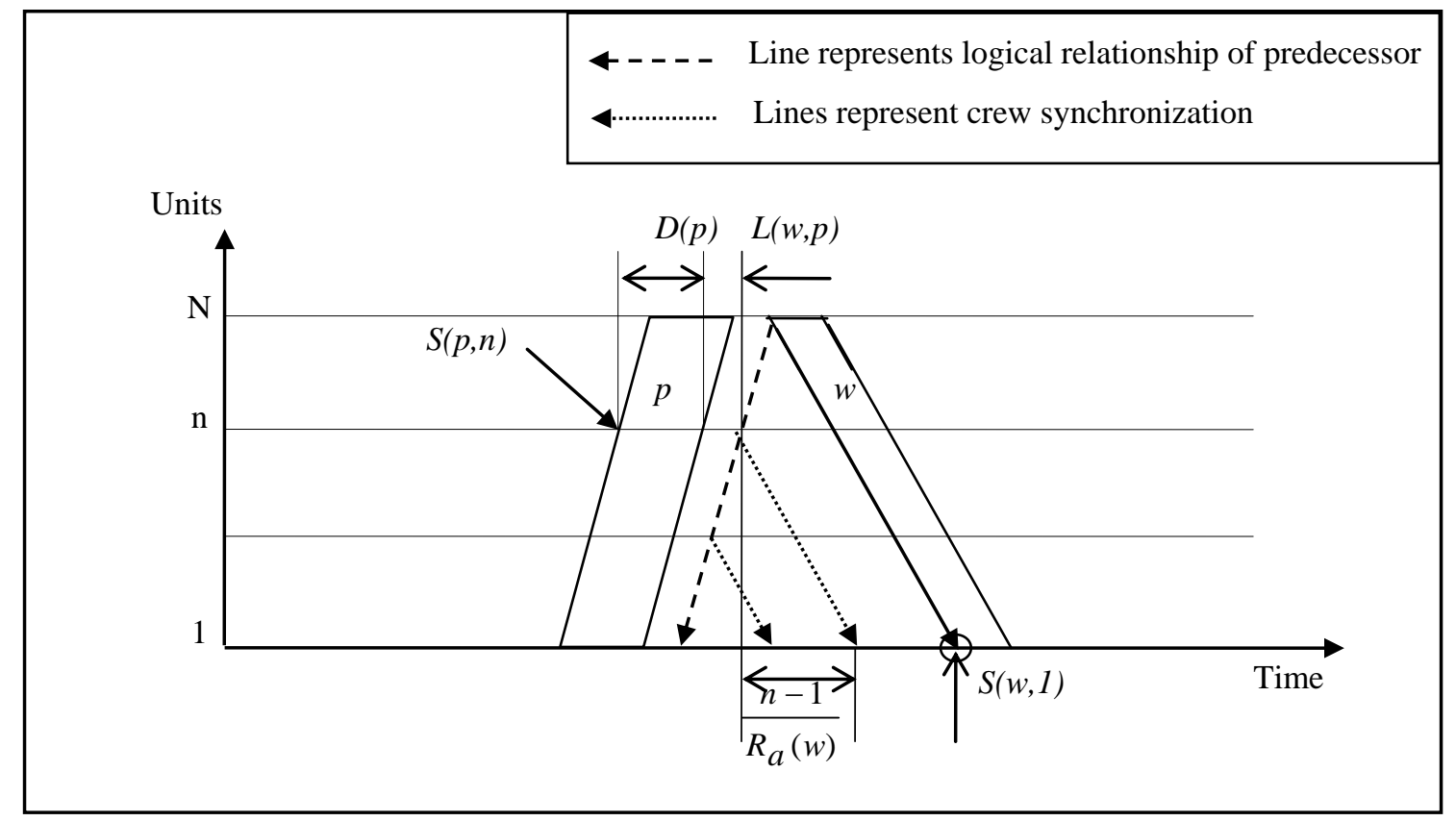

Figure 7. Scheduling downward Non-skeleton Activities at First Unit 


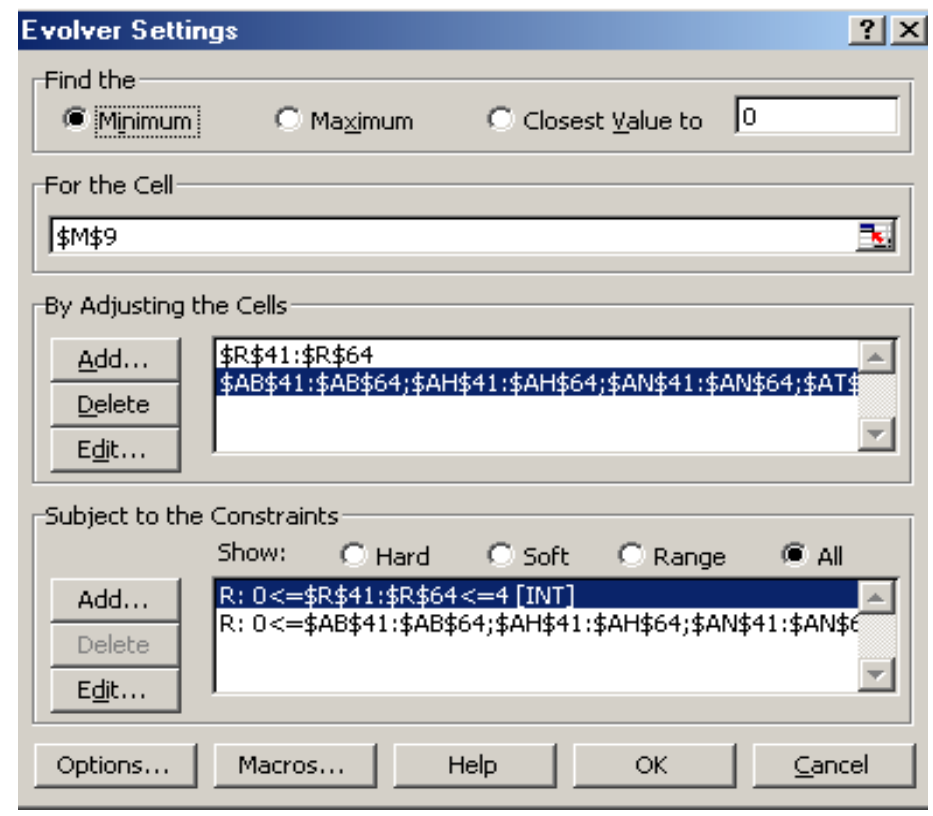

Figure 9. Evolver optimization screen 


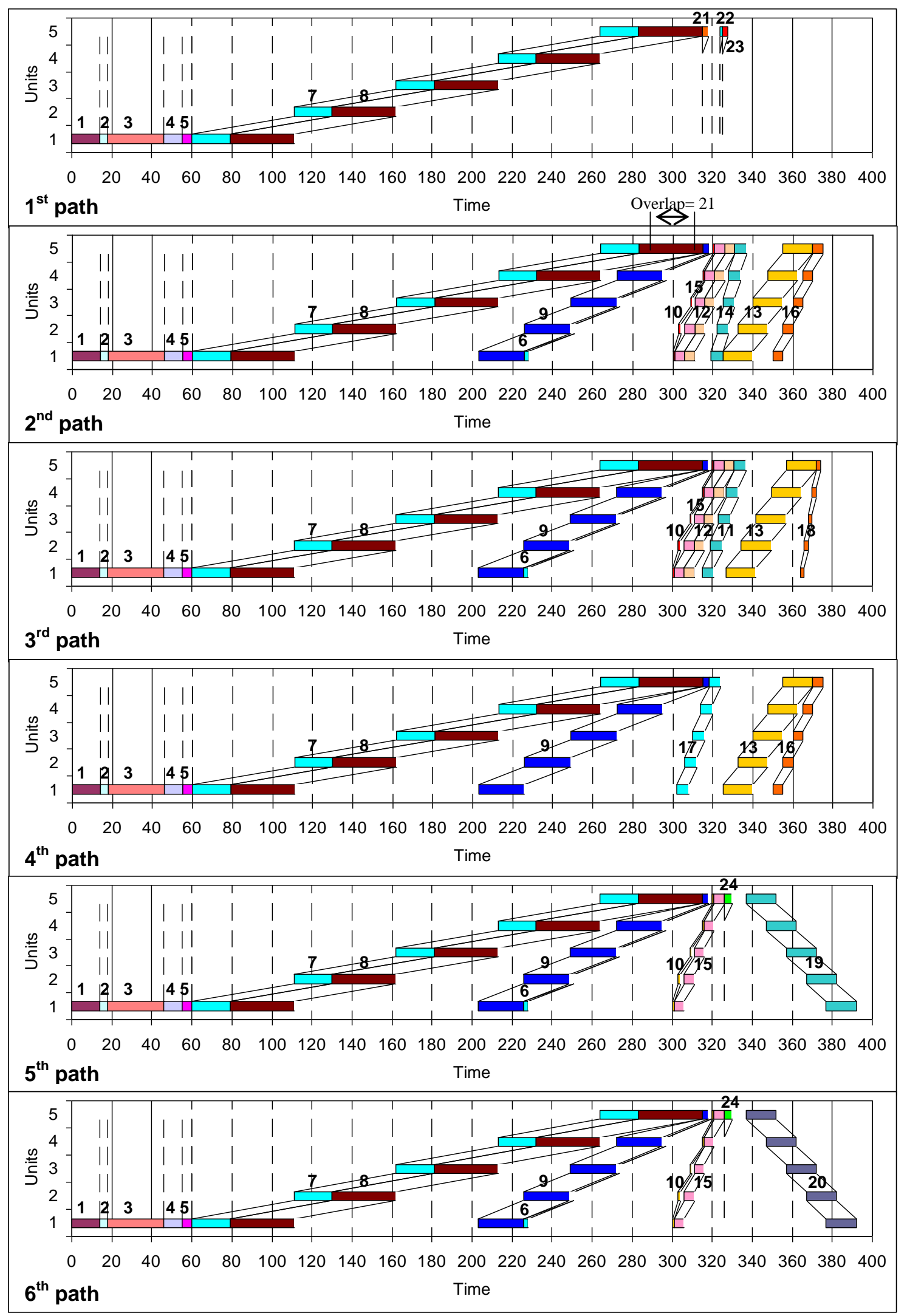

Figure 10. LOB Chart for the example application 
Table 1: Effect of Changing Weights' Values on Schedule Performance

\begin{tabular}{|c|c|c|c|c|c|c|c|c|}
\hline \multicolumn{5}{|c|}{ Scenario } & \multicolumn{3}{|c|}{ Goals } & \multirow{2}{*}{$M O F$} \\
\hline No. & $w_{d}$ & $w_{c}$ & $w_{i}$ & Runs & $P D$ & $\sum C_{a}(i)$ & $\sum I N_{a}(i, n)$ & \\
\hline 1 & 1 & 0 & 0 & $\begin{array}{l}1 \\
2 \\
3 \\
\end{array}$ & 385 & $\begin{array}{l}32 \\
35 \\
36 \\
\end{array}$ & $\begin{array}{l}34 \\
26 \\
30 \\
\end{array}$ & 0.9322 \\
\hline 2 & 0.9 & 0.05 & 0.05 & $\begin{array}{l}1 \\
2 \\
3 \\
\end{array}$ & 385 & $\begin{array}{l}29 \\
29 \\
29 \\
\end{array}$ & $\begin{array}{l}6 \\
6 \\
7 \\
\end{array}$ & $\begin{array}{l}0.8624 \\
0.8624 \\
0.8625 \\
\end{array}$ \\
\hline 3 & 0.8 & 0.1 & 0.1 & $\begin{array}{l}1 \\
2 \\
3 \\
\end{array}$ & 385 & $\begin{array}{l}26 \\
26 \\
26 \\
\end{array}$ & $\begin{array}{l}17 \\
17 \\
16 \\
\end{array}$ & $\begin{array}{l}0.7905 \\
0.7905 \\
0.7903 \\
\end{array}$ \\
\hline 4 & 0.7 & 0.15 & 0.15 & $\begin{array}{l}1 \\
2 \\
3 \\
\end{array}$ & 385 & 26 & 16 & 0.7193 \\
\hline 5 & 0.6 & 0.2 & 0.2 & $\begin{array}{l}1 \\
2 \\
3 \\
\end{array}$ & 385 & 26 & 16 & 0.6483 \\
\hline 6 & 0.5 & 0.25 & 0.25 & $\begin{array}{l}1 \\
2 \\
3\end{array}$ & 385 & 26 & 16 & 0.5773 \\
\hline 7 & 0.4 & 0.3 & 0.3 & $\begin{array}{l}1 \\
2 \\
3 \\
\end{array}$ & 389 & 25 & 16 & 0.5056 \\
\hline 8 & 0.5 & 0.2 & 0.3 & $\begin{array}{l}1 \\
2 \\
3 \\
\end{array}$ & $\begin{array}{l}387 \\
387 \\
385 \\
\end{array}$ & $\begin{array}{l}28 \\
28 \\
26 \\
\end{array}$ & $\begin{array}{c}6 \\
6 \\
16 \\
\end{array}$ & $\begin{array}{l}0.5604 \\
0.5604 \\
0.5590 \\
\end{array}$ \\
\hline 9 & 0.5 & 0.3 & 0.2 & $\begin{array}{l}1 \\
2 \\
3 \\
\end{array}$ & $\begin{array}{l}385 \\
389 \\
389 \\
\end{array}$ & $\begin{array}{l}26 \\
25 \\
25 \\
\end{array}$ & $\begin{array}{l}16 \\
16 \\
16 \\
\end{array}$ & $\begin{array}{l}0.5957 \\
0.5959 \\
0.5959 \\
\end{array}$ \\
\hline
\end{tabular}

